PROCEEDINGS OF THE

AMERICAN MATHEMATICAL SOCIETY

Volume 132, Number 11, Pages 3433-3439

S $0002-9939(04) 07472-6$

Article electronically published on May 20, 2004

\title{
HEREDITARY D-PROPERTY OF FUNCTION SPACES OVER COMPACTA
}

\author{
RAUSHAN Z. BUZYAKOVA \\ (Communicated by Alan Dow) \\ To my teacher Alexander Arhangel'skii for his $65^{\text {th }}$ birthday
}

\begin{abstract}
It is shown that if $X$ is compact then every subspace of $C_{p}(X)$ is a $D$-space in the sense of E. van Douwen, which positively answers Matveev's question. A connection between the $D$-property and Baturov's and Grothendieck's classical theorems about function spaces over compacta is established.
\end{abstract}

\section{INTRODUCTION}

In this paper we will show that for a compactum $X$, every subspace of $C_{p}(X)$ is a $D$-space. This result positively answers Matveev's question whether the conclusion in the Baturov theorem [BAT] for compacta can be strengthened to the $D$-property. Recall that for a compactum $X$, Baturov's theorem states that $l(Y)=e(Y)$ for every $Y \subset C_{p}(X)$. In the above equality $l(Y)$ stands for the Lindelö number, that is, the smallest infinite cardinal $\tau$ such that every open covering of $Y$ contains a subcovering of cardinality $\leq \tau$. Also, $e(Y)$ is the extent of $Y$, defined as the suppremum of cardinalities of closed discrete subsets.

The notion of $D$-space was introduced by Eric van Douwen DOU.

A neighborhood assignment for a space $X$ is a function $\varphi$ from $X$ to the topology of $X$ such that $x \in \varphi(x)$ for any $x \in X$. A space $X$ is a $D$-space, if for any neighborhood assignment $\varphi$ for $X$ there exists a closed discrete subset $D$ of $X$ such that $X=\bigcup_{d \in D} \varphi(d)$.

One can easily prove from the definition that $l(X)=e(X)$ for every $D$-space $X$. Thus, our theorem implies the Baturov theorem for compacta. Also a corollary to our result is the Grothendieck theorem GRO for compacta. This theorem states that if $X$ is compact then every countably compact subset of $C_{p}(X)$ is compact. Other corollaries and some questions will be discussed in the last section of this work. In short, our result widens the class of spaces that are known to be $D$, that is, the $D$-property once again appears at the end of the statement. Unfortunately, we still know very little about how the $D$-property affects a space. Thus, more statements in the form "If $X$ has the D-property then ..." would improve our understanding of this puzzling notion.

Received by the editors April 21, 2003 and, in revised form, July 31, 2003.

2000 Mathematics Subject Classification. Primary 54C35, 54D20, 54C60.

Key words and phrases. $C_{p}(X), D$-space.

The author's research was supported by PSC-CUNY grant 64457-00 33. 
It is known that $\sigma$-compact spaces, metrizable spaces, semi-stratifiable spaces, and paracompact p-spaces are all $D$-spaces (see [DCA], [B\&W1]). In [BUZ], it is shown that every strong $\Sigma$-space is a $D$-space. The $D$-property of subspaces of generalized ordered spaces is studied in [D\&L]. In a recent paper [F\&S] of Fleissner and Stanley, the authors give conditions under which a subspace of a product of finitely many ordinals is a $D$-space. In $\mathrm{A} \& \mathrm{~B}$, the authors obtain some addition theorems for $D$-spaces. Several interesting questions on $D$-spaces were raised by E. van Douwen and W.F. Pfeffer in $[\mathrm{D} \& \mathrm{P}]$. Some other results and questions on $D$-spaces can be also found in [B\&W2], [B\&W3].

Two of the most intriguing questions about $D$-spaces raised by van Douwen are: Is every Lindelöf space a D-space? Is every paracompact space a D-space? These questions remain unanswered.

In notation and terminology we will follow $[\mathrm{ARH}]$ and $[\mathrm{ENG}$. Throughout the paper all spaces are assumed Tychonoff, and by $R$ we denote the space of all reals endowed with the standard topology.

\section{MAIN RESUlt}

Definition 2.1. Let $\mathcal{U}$ be a family of open sets in a space $X$. A family $\mathcal{V}$ is called a finite extension of $\mathcal{U}$ if it is the smallest family with the following properties:

(1) All finite unions of elements of $\mathcal{U}$ are in $\mathcal{V}$.

(2) $X \backslash \overline{\mathcal{U}^{\prime}} \in \mathcal{V}$ for any finite $\mathcal{U}^{\prime} \subset \mathcal{U}$.

Notice that for an infinite $\mathcal{U}$ its finite extension has the same cardinality as $\mathcal{U}$.

Definition 2.2. Let $A \subset Y \subset C_{p}(X)$, and let $\mathcal{B}$ be a base of $R$. Define a family $\mathcal{G}_{A, Y, \mathcal{B}}$ as follows: $G \in \mathcal{G}_{A, Y, \mathcal{B}}$ iff there exist $B_{1}, \ldots, B_{n} \in \mathcal{B}$ and $S_{1}, \ldots, S_{n}$ in the finite extension of $\left\{a^{-1}(B): a \in A, B \in \mathcal{B}\right\}$ such that $G=\left\{f \in Y: f\left(S_{i}\right) \subset B_{i}, i \leq n\right\}$. If it is understood which base $\mathcal{B}$ is under consideration, we write $\mathcal{G}_{A, Y}$.

Notice that for an infinite $A$ and countable $\mathcal{B}$, the cardinality of $\mathcal{G}_{A, Y, \mathcal{B}}$ does not exceed that of $A$. If $A$ is finite, then $\mathcal{G}_{A, Y, \mathcal{B}}$ is countable.

Lemma 2.3. Let $X$ be compact and $Y \subset C_{p}(X)$. Let $\mathcal{B}$ be a base of $R$. Let $f$ be a limit point of $A \subset Y$ and $\phi(f)$ an open neighborhood of $f$ in $Y$. Then there exist a finite $A^{\prime} \subset A$ and $G \in \mathcal{G}_{A^{\prime}, Y}$ such that $f \in G \subset \phi(f)$.

Proof. Let $\mathcal{S}$ be a finite extension of the family $\left\{a^{-1}(B): a \in A, B \in \mathcal{B}\right\}$. Without loss of generality, for some $x_{1}, \ldots, x_{n} \in X$ and $B_{1}, \ldots, B_{n} \in \mathcal{B}, \phi(f)=\{g \in Y$ : $\left.g\left(x_{i}\right) \in B_{i}, i \leq n\right\}$. Let us show that for each $i \leq n$, there exists $S_{i} \in \mathcal{S}$ such that $x_{i} \in S_{i}$ and $f\left(S_{i}\right) \subset B_{i}$.

Take an arbitrary $i \leq n$ and fix it. For each $y \in X$ such that $f(y) \notin B_{i}$ let us fix $B_{y} \in \mathcal{B}$ such that $f\left(x_{i}\right) \notin \overline{B_{y}}$ and $f(y) \in B_{y}$. Also for each such $y$, fix $a_{y} \in A$ such that $a_{y}\left(x_{i}\right) \in B_{i} \backslash \overline{B_{y}}$ and $a_{y}(y) \in B_{y}$ (such an $a_{y}$ exists for each $y$, since $f$ is a limit point for $A$ ). The family $\left\{a_{y}^{-1}\left(B_{y}\right): f(y) \notin B_{i}\right\}$ covers $X \backslash f^{-1}\left(B_{i}\right)$. Since the latter is compact, there exist $a_{y_{1}}^{-1}\left(B_{y_{1}}\right), \ldots, a_{y_{k}}^{-1}\left(B_{y_{k}}\right)$ whose union covers $X \backslash f^{-1}\left(B_{i}\right)$. Put $S_{i}=X \backslash \overline{a_{y_{1}}^{-1}\left(B_{y_{1}}\right) \cup \ldots \cup a_{y_{k}}^{-1}\left(B_{y_{k}}\right)}$. By the definition of a finite extension, $S_{i}$ is an element of a finite extension of the set $\left\{a^{-1}(B): B \in \mathcal{B}, a \in A_{i}\right\}$, where $A_{i}=\left\{a_{y_{1}}, \ldots, a_{y_{k}}\right\}$. By the choice of $B_{y}$ 's and $a_{y}$ 's, we have $x_{i} \in S_{i}$ and $f\left(S_{i}\right) \subset B_{i}$.

Thus, $f \in\left\{g \in Y: g\left(S_{i}\right) \subset B_{i}, i \leq n\right\} \subset \phi(f)$. The middle set in this inclusion belongs to $\mathcal{G}_{A^{\prime}, Y}$, where $A^{\prime}=A_{1} \cup \ldots \cup A_{n}$. 
Theorem 2.4. Let $X$ be compact and $Y \subset C_{p}(X)$. Then $Y$ is a D-space.

Proof. Let $\mathcal{B}$ be a countable base of $R$. Let $\phi$ be a neighborhood assignment for $Y$. We need to construct a closed discrete $D \subset Y$ such that $Y=\bigcup_{d \in D} \phi(d)$.

For each $\alpha \geq \omega$, we will define a Machine ${ }_{\alpha}$ that takes a certain input and produces the following product with a certain property.

Input: a set $A \subset Y$ of cardinality not exceeding $|\alpha|$ and an open $U$ containing $\bigcup_{a \in A} \phi(a)$.

Product: a closed discrete $\mathrm{M}_{\alpha}(A)$ contained in $Y \backslash U$ of cardinality not greater than that of $\alpha$.

Property: If $G \in \mathcal{G}_{A \cup \mathrm{M}_{\alpha}(A), Y}$ and $f \in G \subset \phi(f)$ for some $f \in Y$, then $f \in$ $\left(\bigcup_{a \in A \cup \mathrm{M}_{\alpha}(A)} \phi(a)\right) \cup U$.

At the same step $\alpha$, once Machine is $_{\alpha}$ operating we apply it to define a discrete closed set $D_{\alpha}$ so that $D$ will be $\bigcup D_{\alpha}$.

\section{Construction.}

Step $\omega$.

Machine $_{\omega}$. Take an arbitrary set $A \subset Y$ of cardinality not exceeding $\omega$ and an open $U \subset Y$ containing $\bigcup_{a \in A} \phi(a)$. If $U$ covers $Y$, then $\mathrm{M}_{\omega}(A)=\emptyset$ has the required Property, and we are done.

If we are not that lucky, we will have to perform an inductive construction to produce $\mathrm{M}_{\omega}(A)=\left\{a_{n}: n>1\right\}$. Starting from Substep 2 we will follow our induction using elements of the set $\mathcal{G}_{A, Y}$. However, at each Substep $n$, we might need to enrich $\mathcal{G}_{A, Y}$ by countably many new elements. To make sure that we are not lost among new elements, we agree to enumerate $\mathcal{G}_{A, Y}$ by prime numbers, while new elements added at Substep $n$ are enumerated by numbers $p^{n+1}$, where $p$ is any prime. Notice that this method of enumeration guarantees that once an element is tagged with an integer $m$, it keeps the tag even when new elements are added.

Substep 1. Let $A_{1}=A$. Enumerate elements of $\mathcal{G}_{A_{1}, Y}$ by prime numbers.

Substep $n$. Take the first $G \in \mathcal{G}_{A_{n-1}, Y}$ that satisfies the following requirement.

Requirement: There exists $a_{n} \in Y \backslash\left[\left(\bigcup_{a \in A_{n-1}} \phi(a)\right) \cup U\right]$ such that $a_{n} \in G \subset \phi\left(a_{n}\right)$.

Put $A_{n}=A_{n-1} \cup\left\{a_{n}\right\}$. Enumerate elements of $\mathcal{G}_{A_{n}, Y}$ in such a manner that elements of $\mathcal{G}_{A_{n}, Y} \backslash \mathcal{G}_{A_{n-1}, Y}$ are tagged with numbers $p^{n+1}$, where $p$ is any prime, while enumeration on $\mathcal{G}_{A_{n-1}, Y}$ is left unchanged.

If no such $G$ exists, stop the construction.

Let $\mathrm{M}_{\omega}(A)=\left\{a_{n}: n>1\right\}$. First notice that $\mathrm{M}_{\omega}(A) \subset Y \backslash U$ holds, as all $a_{n}$ 's are taken from the complement of $U$. Let us show that $\mathrm{M}_{\omega}(A)$ has the Property. Let $f \in G \subset \phi(f)$, for some $G \in \mathcal{G}_{A \cup \mathrm{M}_{\omega}(A), Y}$. Then there exists a smallest $n \in N$ such that $G \in \mathcal{G}_{A_{n}, Y}$. If $f$ is not covered by $\left(\bigcup_{a \in A_{n}} \phi(a)\right) \cup U$, starting from Substep $n+1$, the element $G$ satisfies the Requirement. Therefore $G$ must be covered, and so must $f$.

Let us show that $\mathrm{M}_{\omega}(A)$ is closed and discrete in $Y$. Assume the contrary. Then there exists $f \in Y$, a limit point for $\mathrm{M}_{\omega}(A)$. By Lemma 2.3, there exists $n \in N$ such that $f \in G \subset \phi(f)$ for some $G \in \mathcal{G}_{A_{n}, Y}$. Therefore, by Property, $f$ must be covered by $\left(\bigcup_{a \in A_{m}} \phi(a)\right) \cup U$ at some Substep $m$. This means $f$ is separated from all $a_{i}$ 's, where $i>m$, by $\left(\bigcup_{a \in A_{m}} \phi(a)\right) \cup U$. It is also separated from the rest of the $a_{i}$ 's (distinct from $f$ ), as there are finitely many of them. 
Construction of $D_{\omega}$. Take an arbitrary $d \in Y$. Apply Machine $\omega$ to $A=\{d\}$ and $\overline{U=\phi(d) \text { to generate }} \mathrm{M}_{\omega}(A)$. Put $D_{\omega}=\mathrm{M}_{\omega}(A) \cup\{d\}$.

Assumption. Assume that for all $\beta<\alpha$, Machine $\beta$ is constructed, and $D_{\beta}$ is defined and is of cardinality not greater than $|\beta|$. Assume also that if $\beta_{1}<\beta_{2}$, then $D_{\beta_{1}} \subset D_{\beta_{2}}$ and $D_{\beta_{2}} \backslash D_{\beta_{1}} \subset Y \backslash \bigcup_{d \in D_{\beta_{1}}} \phi(d)$.

Step $\alpha$.

Machine $_{\alpha}$. If $|\alpha|=|\beta|$ for some $\beta<\alpha$, then Machine $\beta$ does everything we need. Otherwise, the cardinality of $\alpha$ is greater than that of any smaller ordinal number. Take an arbitrary set $A \subset Y$ of cardinality not exceeding $|\alpha|$ and an open set $U$ containing $\bigcup_{a \in A} \phi(a)$. If $U$ covers $Y$, then put $\mathrm{M}_{\alpha}(A)=\emptyset$ and we are done. Otherwise, enumerate $A$ by ordinals less than $\alpha$. We can assume that $|A|=|\alpha|$, since for smaller $A$ 's, machines with smaller tags can do the job.

Inductively, for each $\omega \leq \gamma<\alpha$, we will define a closed discrete $A_{\gamma}$ and $\mathrm{M}_{\alpha}(A)$ will be set equal to $\bigcup_{\omega \leq \gamma<\alpha} A_{\gamma}$.

Substep $\omega$. Apply Machine $\omega_{\omega}$ to the set $B_{\omega}=\left\{a_{n}: n<\omega\right\}$ and $U_{1}=U$ to generate $A_{\omega}=\mathrm{M}_{\omega}\left(B_{\omega}\right)$.

Assumption. Assume that a closed discrete $A_{\beta} \subset Y \backslash U$ is defined for every $\beta<\gamma$ and is of cardinality not greater than $|\beta|$. Also assume that if $\beta_{1}<\beta_{2}$, then $A_{\beta_{1}} \subset A_{\beta_{2}}$ and $A_{\beta_{2}} \backslash A_{\beta_{1}} \subset Y \backslash \bigcup_{a \in A_{\beta_{1}}} \phi(a)$.

Substep $\gamma<\alpha$. If $\bigcup\left\{\phi(a): a \in A_{\beta}, \beta<\gamma\right\} \cup U$ covers $Y$, stop the construction.

Since $\left|A_{\beta}\right| \leq|\beta|$ for all $\beta<\gamma$, the cardinality of $B_{\gamma}=\bigcup_{\beta<\gamma} A_{\beta} \cup$ $\left\{a_{\beta} \in A: \beta<\gamma\right\}$ equals $|\gamma|$. Apply Machine $_{\gamma}$ to the set $B_{\gamma}$ and open $U_{\gamma}=\bigcup\left\{\phi(a): a \in A_{\beta}\right.$ for $\left.\beta<\gamma\right\} \cup U$ to generate $\mathrm{M}_{\gamma}\left(B_{\gamma}\right)$. Put $A_{\gamma}=\mathrm{M}_{\gamma}\left(B_{\gamma}\right) \cup\left(\bigcup_{\beta<\gamma} A_{\beta}\right)$. Clearly $\left|A_{\gamma}\right| \leq|\gamma|$. The inclusions in the induction assumption hold by construction. Finally, $A_{\gamma}$ is closed and discrete because $\mathrm{M}_{\gamma}\left(B_{\gamma}\right)$ is such by assumption while $\bigcup_{\beta<\gamma} A_{\beta}$ is closed and discrete by the following Remark.

Remark. The set $\bigcup_{\beta<\gamma} A_{\beta}$ is closed and discrete in $Y$. Assume this set has a limit point $f$. Then by Lemma 2.3, there exists a finite $A^{\prime} \subset \bigcup_{\beta<\gamma} A_{\beta}$ such that $f \in G \subset \phi(G)$ for some $G \in \mathcal{G}_{A^{\prime}, Y}$. By assumption, there exists $\beta<\gamma$ such that $A^{\prime} \subset A_{\beta}$. Therefore, by Property, $f$ is covered by $\left(\bigcup_{a \in A_{\beta}} \phi(a)\right) \cup U$. We may assume that $f$ is covered for the first time at Substep $\beta$ by $\phi\left(a^{*}\right)$. By the construction assumption, $\phi\left(a^{*}\right)$ separates $f$ from all $a$ 's chosen after Substep $\beta$. And $f$ cannot be a limit for the $A_{\beta}$, as the latter is closed and discrete by assumption.

Let $\mathrm{M}_{\alpha}(A)=\bigcup_{\beta<\alpha} A_{\beta}$. Since $\left|A_{\beta}\right| \leq \beta$, we have $\left|\mathrm{M}_{\alpha}(A)\right| \leq \alpha$. By the remark, $\mathrm{M}_{\alpha}(A)$ is closed and discrete in $Y$. Since all $A_{\beta}$ 's are in the complement of $U$, our set is there as well. Let us show that $\mathrm{M}_{\alpha}(A)$ has the Property. Take any $G \in \mathcal{G}_{A \cup \mathrm{M}_{\alpha}(A), Y}$ such that $f \in G \subset \phi(f)$ for some $f \in Y$. By the definition of the family $\mathcal{G}_{A \cup \mathrm{M}_{\alpha}(A), Y}$, there exists a finite $A^{\prime} \subset A \cup \mathrm{M}_{\alpha}(A)$ such that $G \in \mathcal{G}_{A^{\prime}, Y}$. Therefore, there exists $\gamma<\alpha$ such that $G \in \mathcal{G}_{\left\{a_{\beta} \in A: \beta<\gamma\right\} \cup A_{\gamma}, Y}$. Applying the inductive definition of $A_{\gamma}$ and the Property of $\mathrm{M}_{\gamma}\left(B_{\gamma}\right)$, we have $f \in \bigcup_{a \in A_{\gamma}} \phi(a) \cup U$. This inclusion proves that $\mathrm{M}_{\alpha}(A)$ has the Property. 
Construction of $D_{\alpha}$. First notice that by the remark, $\bigcup_{\beta<\alpha} D_{\beta}$ is closed and discrete in $Y$. Now, take an arbitrary $d_{\alpha} \in Y \backslash \cup\left\{\phi(d): d \in D_{\beta}, \beta<\alpha\right\}$. Apply Machine $_{\alpha}$ to the closed discrete set $A=\left(\bigcup_{\beta<\alpha} D_{\beta}\right) \cup\left\{d_{\alpha}\right\}$ and the open set $U=\bigcup\left\{\phi(d): d \in D_{\beta}, \beta<\alpha\right.$ or $\left.d=d_{\alpha}\right\}$ to generate $\mathrm{M}_{\alpha}(A)$. Put $D_{\alpha}=$ $\mathrm{M}_{\alpha}(A) \cup\left(\bigcup_{\beta<\alpha} D_{\beta}\right) \cup\left\{d_{\alpha}\right\}$.

If $Y=\bigcup\left\{\phi(d): d \in D_{\beta}, \beta<\alpha\right\}$, stop the construction.

Let $D=\bigcup_{\alpha} D_{\alpha}$. The set $\bigcup_{d \in D} \phi(d)$ covers $Y$, since we continue our construction until $Y$ is covered. And it is closed and discrete by the remark.

\section{Corollaries and Related problems}

The definition of a $D$-space implies that $l(X)=e(X)$ for every $D$-space $X$. This simple observation leads us to the following corollaries, which are, in fact, well-known classical theorems.

Corollary 3.1 (Baturov's Theorem for compacta). Let $X$ be compact. Then $l(Y)=$ $e(Y)$ for every subspace $Y$ of $C_{p}(X)$.

Corollary 3.2 (Grothendieck's Theorem for Compacta). Let $X$ be compact and $Y$ a countably compact subspace of $C_{p}(X)$. Then $Y$ is compact.

It is known that the $D$-property is preserved by closed continuous maps (see [B\&W1]). Therefore, the following generalizations of Grothendieck's and Baturov's theorems hold.

Corollary 3.3. Let $X$ be compact and $Z$ a closed continuous image of a subspace of $C_{p}(X)$. Then $l(Y)=e(Y)$ for every $Y \subset Z$.

Corollary 3.4. Let $X$ be compact and $Z$ a closed continuous image of a subspace of $C_{p}(X)$. Then every countably compact $Y \subset Z$ is compact.

In fact, the Baturov theorem in question holds not only for compacta but for all Lindelöf $\Sigma$-spaces. Thus, the following question might have a chance for an affirmative answer.

Question 3.5. Let $X$ be a Lindelöf $\Sigma$-space. Is it true that $C_{p}(X)$ is a hereditary $D$-space?

Since $l(X)=e(X)$ for every $D$-space $X$, one might wonder if the reverse implication is true. The unsurprising answer is "No". For a counterexample, consider $X=D\left(\omega_{1}\right) \times \omega_{1}$, where $D\left(\omega_{1}\right)$ is a discrete space of cardinality $\omega_{1}$. A closed copy of $\omega_{1}$ in $X$ makes the space non- $D$, while it is easy to see that $l(X)=e(X)=\omega_{1}$. Yet the latter equality does not hold for every subspace of $X$. Thus, the following question might be of interest.

Question 3.6. Suppose that $l(Y)=e(Y)$ for every subspace $Y$ of a space $X$. Is $X$ then a hereditary $D$-space?

And here are two more quite natural questions related to the Baturov theorem.

Question 3.7 ( $D$-version of Reznichenko's question). Let $X$ be a countably compact space. Is it true that every subspace of $C_{p}(X)$ is a $D$-space?

Question 3.8 (A. Arhangelskii). Let $X$ be compact and $Y$ a separable metrizable space. Is $C_{p}(X \oplus Y)$ then a hereditary $D$-space? 
Recall that any Eberlein compactum is a subspace of $C_{p}(X)$ for some compactum $X$, while a Corson compactum is defined as a compact subspace of a $\Sigma$-product of a family of closed intervals.

Corollary 3.9. Any Eberlein compactum is a hereditary D-space.

Question 3.10 (A. Arhangelskii). Is it true that any Corson compactum is a hereditary $D$-space?

And, let us finish with two more corollaries about function spaces over $\sigma$-compact spaces. Recall that if a space $X$ is $\sigma$-compact then $C_{p}(X)$ is homeomorphic to a subspace of $C_{p}(Y)$ for some compactum $Y$. Also recall that $C_{p}\left(\Sigma_{\oplus}\left\{X_{n}: n \in \omega\right\}\right)$ is homeomorphic to $\Pi\left\{C_{p}\left(X_{n}\right): n \in \omega\right\}$. (Proofs for these two facts can be found in ARH. .) These two facts together with our main result lead to the following observations.

Corollary 3.11. Let $X$ be a $\sigma$-compact space. Then $C_{p}(X)$ is a hereditary $D$-space.

Corollary 3.12. Let $X$ be compact. Then $\left(C_{p}(X)\right)^{n}$ and $\left(C_{p}(X)\right)^{\omega}$ are hereditary D-spaces.

\section{AFTER-SUBMISSION REMARKS}

After this paper was submitted, G. Gruenhage showed that Lemma 2.3 holds for $\Sigma$-Lindelöf spaces as well. And since the argument of the theorem needs from $X$ nothing more than the conclusion of Lemma 2.3, the theorem holds for $\Sigma$-Lindelöf spaces. As a consequence of Gruenhage's result, the answer to Questions 3.5 and 3.8 is "Yes". Also, Gruenhage showed that any Corson compactum is hereditarily a D-space, which settles Question 3.10. The author recently answered Question 3.7 in the negative by constructing an example of a countably compact space $X$ such that $l\left(C_{p}(X)\right) \neq e\left(C_{p}(X)\right)$. In connection with this example, it might be worth mentioning that Theorem 2.4 nevertheless holds for some countably compact spaces.

Theorem 3.13. Let $X$ be a countably compact space such that every open cover of $X$ of cardinality less than $n w(X)$ contains a finite subcover. Then $C_{p}(X)$ is hereditarily a D-space.

Recall that the netweight $n w(X)$ of a space $X$ is defined as the minimum of cardinalities of networks for $X$, where a network is the same as a base but sets need not be open. To see why the above theorem holds, first notice that if in Lemma 2.3 the space $X$ satisfies the hypothesis of Theorem 3.13 and $|A|<n w(X)$, then the lemma's conclusion still holds. And how can we use this new version of Lemma 2.3? The argument of Theorem 2.4 is valid as long as Lemma 2.3 is true. And the new version of the lemma is applicable as long as we are below level $\alpha=n w(X)$. So to redo the proof, we have to make sure that the space is covered by the time we hit level $n w(X)$. And this is easily achieved as follows:

(1) Enumerate a fixed network $\mathcal{F}$ of $Y \subset C_{p}(X)$ by ordinals less than $n w(X)$ (recall that $n w(X)=n w\left(C_{p}(X)\right)$ for any $\left.X\right)$.

(2) At step $\alpha$ in the definition of $D_{\alpha}$ replace "take an arbitrary $d_{\alpha} \ldots$ " by "take the first $F \in \mathcal{F}$ such that there exists a $d_{\alpha} \in Y \backslash \bigcup\left\{\phi(d): d \in D_{\beta}, \beta<\alpha\right\}$ such that $d_{\alpha} \in F \subset \phi\left(d_{\alpha}\right)$." Such an $F$ exists. Indeed, if $Y$ is not covered yet, take an arbitrary $d$ from the uncovered part of $Y$. By the definition of 
a network, there exists an $F$ such that $d \in F \subset \phi(d)$. Now take the first such $F$.

This change guarantees that we cover the space as soon as all network elements are covered (not more than $n w(X)$ steps are needed).

An immediate corollary to Theorem 3.13 is that $C_{p}$ over any countably compact space of weight $\omega_{1}$ is hereditarily a $D$-space. In particular, $C_{p}\left(\omega_{1}\right)$ is such.

\section{ACKNOWLEDGMENT}

The author would like to thank the referee for valuable remarks and suggestions.

\section{REFERENCES}

[ARH] A. Arhangelskii, Topological function spaces, Math. Appl., vol. 78, Kluwer Academic Publisher, Dordrecht, 1992. MR 92i:54022

[A\&B] A. Arhangelskii and R. Buzyakova, Addition theorems and D-spaces, Comment. Math. Universitatis Carolinae 43, 4(2002), 653-663.

[BAT] D. Baturov, On subspaces of function spaces, Vestn. Moskov. Univ. Ser. I Mat. Mech., 1987, no. 4, 66-69; English transl. in Moscow Univ. Math. Bull. 52 (1997). MR 89a:54018

[BUZ] R. Buzyakova, On D-property of strong $\Sigma$-spaces., Comment. Math. Universitatis Carolinae, 43, 3(2002), 493-495. MR 2003j:54021

[B\&W1] C.R. Borges and A.C. Wehrly, A study of D-spaces. Topology Proc. 16 (1991), 7 - 15. MR 94a:54059

[B\&W2] C.R. Borges and A.C. Wehrly, Another study of D-spaces. Questions and Answers in General Topology 14:1 (1996), 73 - 76. MR 96m:54033

[B\&W3] C.R. Borges and A.C. Wehrly, Correction: another study of D-spaces. Questions and Answers in General Topology 16:1 (1998), 77 - 78. MR 98m:54026

[DCA] P. DeCaux, Yet another property of the Sorgenfrey plane, Topology Proc. 6:1 (1981), 31-43. MR 83h:54032

[DOU] E.K. van Douwen, Simultaneous extension of continuous functions. Thesis, Free University, Amsterdam, 1975. Cf. MR 52:1612

[D\&L] E. K. van Douwen and D. J. Lutzer, A note on paracompactness in generalized ordered spaces, Proc. AMS, 125(1997), 1237-1245. MR 97f:54039

[D\&P] E.K. van Douwen and Washek F. Pfeffer, Some properties of the Sorgenfrey line and related spaces. Pacific Journ. of Math. 81 (1979), 371 - 377. MR 80h:54027

[ENG] R. Engelking, General Topology, Sigma Series in Pure Mathematics, 6, Heldermann, Berlin, revised ed., 1989. MR 91c:54001

[F\&S] W. G. Fleissner and A. M. Stanley, D-spaces, Topology and Appl., 114(2001), 261-271. MR 2002e:54013

[GRO] A. Grothendieck, Critères de compacité dans les espaces fonctionnels généraux, Amer. J. Math. (74) (1952), 168-186. MR 13:857e

Department of Mathematics, Brooklyn College, Brooklyn, New York 11210

E-mail address: RaushanB@brooklyn.cuny.edu 